\title{
Scale-free Linear Observer-based Protocol Design for Global Regulated State Synchronization of Homogeneous Multi-agent Systems with Non-introspective Agents Subject to Input Saturation
}

\author{
Zhenwei Liu ${ }^{1}$, Donya Nojavanzadeh ${ }^{2}$, Ali Saberi ${ }^{2}$, Anton A. Stoorvogel ${ }^{3}$ \\ 1. College of Information Science and Engineering, Northeastern University, Shenyang 110819, China \\ E-mail: liuzhenwei@ise.neu.edu.cn \\ 2. School of Electrical Engineering and Computer Science, Washington State University, Pullman, WA 99164, USA \\ E-mail: donya.nojavanzadeh@wsu.edu; saberi@wsu.edu \\ 3. Department of Electrical Engineering, Mathematics and Computer Science, University of Twente, Enschede, The Netherlands \\ E-mail: A.A.Stoorvogel@utwente.nl
}

\begin{abstract}
This paper studies global regulated state synchronization of homogeneous networks of non-introspective agents in presence of input saturation. We identify three classes of agent models which are neutrally stable, doubleintegrator, and mixed of double-integrator, single-integrator and neutrally stable dynamics. A scale-free linear observerbased protocol design methodology is developed based on localized information exchange among neighbors where the reference trajectory is given by a so-called exosystem which is assumed to be globally reachable. Our protocols do not need any knowledge about the communication network topology and the spectrum of associated Laplacian matrix. Moreover, the proposed protocol is scalable and is designed based on only knowledge of agent models and achieves synchronization for any communication graph with arbitrary number of agents.
\end{abstract}

Key Words: Multi-agent systems, Global regulated state synchronization, Scale-free protocol design

\section{Introduction}

The synchronization problem of networks consisting of linear or nonlinear agents has become a hot topic among researchers during the past decade due to the wide potential for applications in several areas such as automotive vehicle control, satellites/robots formation, sensor networks, and so on. The objective of synchronization is to secure asymptotic agreement on a common state or output trajectory by control protocols with local communication information, see for instance the books [19] and [31] or the survey paper [16].

Generally, synchronization of multi-agent system (MAS) includes two main types, state and output synchronization. Because the state synchronization inherently requires homogeneous networks (i.e. agents which have identical dynamics), most work in synchronization for MAS focused on state synchronization of homogeneous networks. State synchronization based on diffusive full-state coupling has been studied where the agent dynamics progress from single- and double-integrator (e.g. [17], [18]) to more general dynamics (e.g. [21], [27],

This work is supported by Nature Science Foundation of Liaoning Province under Grant 2019-MS-116.
[29]). State synchronization based on diffusive partialstate coupling has also been considered, including static design ([12] and [13]), dynamic design ([5], [22], [23], [26], [28]), and the design based on localized information exchange ([1] and [21]). Solvability conditions are studied for general case of full and partial-state coupling in [25], [24]. Recently, scale-free collaborative protocol designs are developed for continuous-time heterogeneous MAS [15] and for homogeneous MAS subject to actuator saturation [10] and subject to input delays [9, 8].

Meanwhile, if the agents have absolute measurements of their own dynamics in addition to relative information from the network, they are said to be introspective, otherwise, they are called non-introspective. There exist some results about these two types of agents, for example, introspective agents $([6,33]$, etc), and non-introspective agents $([4,30]$, etc).

On the other hand, it is worth to note that actuator saturation is pretty common and indeed is ubiquitous in engineering applications. Many researchers have tried to establish (semi) global state and output synchronization results for multi-agent system (MAS) in the presence of input saturation. Compared with semi-global results, global synchronization can work for any initial condi- 
tion set, and thus it has wider applications and attracts more attention. Global synchronization for neutrally stable agents has been studied by [14] (continuous-time) and [32] (discrete-time) for either undirected or detailed balanced graph. Then, global synchronization via static protocols for MAS with partial state coupling and linear general dynamics is developed in [11]. Reference [7] provides the design which can deal with networks that are not detailed balanced but intrinsically requires the agents to be single integrator. Similar scenarios also can be found in [2] (finite-time consensus), and [34] (event-triggered control).

In this paper, we design scale-free linear observerbased dynamic protocols to achieve global regulated state synchronization for homogeneous networks of nonintrospective agents in presence of input saturation utilizing localized information exchange among the neighbors. The contributions of this paper are stated as follow.

- We develop scale-free linear observer-based dynamic protocols for MAS with non-introspective agents and for three classes of agent models which are neutrally stable, double-integrator, and mixed of double-integrator, single-integrator and neutrally stable dynamics and for both networks with fulland partial-state coupling. Moreover, the proposed linear protocols have infinite gain margins.

- Linear observer-based protocol designs are scalefree and do not need any information about communication network. In other words, the proposed protocols work for any MAS with any communication graph with arbitrary number of agents.

\section{Notations and definitions}

Given a matrix $A \in \mathbb{R}^{m \times n}, A^{\mathrm{T}}$ denotes the transpose of $A$ and $\|A\|$ denotes the induced 2-norm of $A$. For a vector $x \in \mathbb{R}^{q},\|x\|$ denotes the 2 -norm of $x$ respectively. A square matrix $A$ is said to be Hurwitz stable if all its eigenvalues are in the open left half complex plane. $A \otimes B$ depicts the Kronecker product between $A$ and $B . I_{n}$ denotes the $n$-dimensional identity matrix and $0_{n}$ denotes $n \times n$ zero matrix; sometimes we drop the subscript if the dimension is clear from the context.

To describe the information flow among the agents we associate a weighted graph $\mathcal{G}$ to the communication network. The weighted graph $\mathcal{G}$ is defined by a triple $(\mathcal{V}, \mathcal{E}, \mathcal{A})$ where $\mathcal{V}=\{1, \ldots, N\}$ is a node set, $\mathcal{E}$ is a set of pairs of nodes indicating connections among nodes, and $\mathcal{A}=\left[a_{i j}\right] \in \mathbb{R}^{N \times N}$ is the weighted adjacency matrix with non negative elements $a_{i j}$. Each pair in $\mathcal{E}$ is called an $e d g e$, where $a_{i j}>0$ denotes an edge $(j, i) \in \mathcal{E}$ from node $j$ to node $i$ with weight $a_{i j}$. Moreover, $a_{i j}=0$ if there is no edge from node $j$ to node $i$. We assume there are no self-loops, i.e. we have $a_{i i}=0$. A path from node $i_{1}$ to $i_{k}$ is a sequence of nodes $\left\{i_{1}, \ldots, i_{k}\right\}$ such that $\left(i_{j}, i_{j+1}\right) \in \mathcal{E}$ for $j=1, \ldots, k-1$. A directed tree is a subgraph (subset of nodes and edges) in which every node has exactly one parent node except for one node, called the root, which has no parent node. The root set is the set of root nodes. A directed spanning tree is a subgraph which is a directed tree containing all the nodes of the original graph. If a directed spanning tree exists, the root has a directed path to every other node in the tree.

For a weighted graph $\mathcal{G}$, the matrix $L=\left[\ell_{i j}\right]$ with

$$
\ell_{i j}=\left\{\begin{array}{c}
\sum_{k=1}^{N} a_{i k}, i=j, \\
-a_{i j}, \quad i \neq j,
\end{array}\right.
$$

is called the Laplacian matrix associated with the graph $\mathcal{G}$. The Laplacian matrix $L$ has all its eigenvalues in the closed right half plane and at least one eigenvalue at zero associated with right eigenvector 1 [3]. Moreover, if the graph contains a directed spanning tree, the Laplacian matrix $L$ has a single eigenvalue at the origin and all other eigenvalues are located in the open right-half complex plane [19].

\section{Problem Formulation}

Consider a MAS consisting of $N$ identical dynamic agents with input saturation:

$$
\left\{\begin{array}{l}
\dot{x}_{i}=A x_{i}+B \sigma\left(u_{i}\right), \\
y_{i}=C x_{i}
\end{array}\right.
$$

where $x_{i} \in \mathbb{R}^{n}, y_{i} \in \mathbb{R}^{q}$ and $u_{i} \in \mathbb{R}^{m}$ are the state, output, and the input of agent $i=1, \ldots, N$, respectively. Meanwhile,

$$
\sigma(v)=\left(\begin{array}{c}
\operatorname{sat}\left(v_{1}\right) \\
\operatorname{sat}\left(v_{2}\right) \\
\vdots \\
\operatorname{sat}\left(v_{m}\right)
\end{array}\right) \text { where } v=\left(\begin{array}{c}
v_{1} \\
v_{2} \\
\vdots \\
v_{m}
\end{array}\right) \in \mathbb{R}^{m}
$$

with $\operatorname{sat}(w)$ is the standard saturation function:

$$
\operatorname{sat}(w)=\operatorname{sgn}(w) \min (1,|w|)
$$

The network provides agent $i$ with the following information,

$$
\zeta_{i}=\sum_{j=1}^{N} a_{i j}\left(y_{i}-y_{j}\right),
$$

where $a_{i j} \geqslant 0$ and $a_{i i}=0$. This communication topology of the network can be described by a weighted graph $\mathcal{G}$ associated with (2), with the $a_{i j}$ being the coefficients 
of the weighted adjacency matrix $\mathcal{A}$. In terms of the coefficients of the associated Laplacian matrix $L, \zeta_{i}$ can be rewritten as

$$
\zeta_{i}=\sum_{j=1}^{N} \ell_{i j} y_{j}
$$

We refer to (3) as partial-state coupling since only part of the states are communicated over the network. When $C=I$, it means all states are shared over the network and we call it full-state coupling.

We also introduce a localized information exchange among neighbors. In particular, each agent $i=1, \ldots, N$ has access to a localized information denoted by $\hat{\zeta}_{i}$, of the form

$$
\hat{\zeta}_{i}=\sum_{j=1}^{N} a_{i j}\left(\xi_{i}-\xi_{j}\right)
$$

where $\xi_{j} \in \mathbb{R}^{n}$ is a variable produced internally by agent $j$ and to be defined in next sections.

In this paper, we consider regulated state synchronization where state of agents converge to a priori given trajectory $x_{r}$ generated by a so-called exosystem

$$
\dot{x}_{r}=A x_{r}, \quad y_{r}=C x_{r} .
$$

with $x_{r} \in \mathbb{R}^{n}$. Clearly, we need some level of communication between the exosystem and the agents. We assume that a nonempty subset $\mathscr{C}$ of the agents have access to their own output relative to the output of the exosystem. Specially, each agent $i$ has access to the quantity

$$
\psi_{i}=\iota_{i}\left(y_{i}-y_{r}\right), \quad \iota_{i}= \begin{cases}1, & i \in \mathscr{C}, \\ 0, & i \notin \mathscr{C} .\end{cases}
$$

Combined with (2), we have the following network exchange

$$
\bar{\zeta}_{i}=\sum_{j=1}^{N} a_{i j}\left(y_{i}-y_{j}\right)+\iota_{i}\left(y_{i}-y_{r}\right) .
$$

$\bar{\zeta}_{i}$, as defined in above, can be rewritten in terms of the coefficients of a so-called expanded Laplacian matrix $\bar{L}=L+\operatorname{diag}\left\{\iota_{i}\right\}=\left[\bar{\ell}_{i j}\right]_{N \times N}$ as

$$
\bar{\zeta}_{i}=\sum_{j=1}^{N} \bar{\ell}_{i j}\left(y_{j}-y_{r}\right) .
$$

Note that $\bar{L}$ is not a regular Laplacian matrix associated to the graph, since the sum of its rows need not be zero. We know that all the eigenvalues of $\bar{L}$, have positive real parts. In particular matrix $\bar{L}$ is invertible.
To guarantee that each agent gets the information from the exosystem, we need to make sure that there exists a path from node set $\mathscr{C}$ to each node. Therefore, we define the following set of graphs.

Definition 1 Given a node set $\mathscr{C}$, we denote by $\mathbb{G}_{\mathscr{C}}^{N}$ the set of all graphs with $N$ nodes containing the node set $\mathscr{C}$, such that every node of the network graph $\mathcal{G} \in \mathbb{G}_{\mathscr{C}}^{N}$ is a member of a directed tree which has its root contained in the node set $\mathscr{C}$. We will refer to the node set $\mathscr{C}$ as root set.

Remark 1 Note that Definition 1 does not require necessarily the existence of directed spanning tree. If the root of the trees belongs to the set $\mathscr{C}$, this means all the agents of the network will have access to the information of the exosystem, i.e. we do not need necessarily the existence of the spanning tree.

Next, we formulate scalable global regulated state synchronization problem with linear protocols.

Problem 1 Consider a MAS described by (1) and (8) and the associated exosystem (5). Let a set of nodes $\mathscr{C}$ be given which defines the set $\mathbb{G}_{\mathscr{C}}^{N}$.

The scalable global regulated state synchronization problem based on localized information exchange of a MAS is to find, if possible, a linear observer-based dynamic protocol for each agent $i \in\{1, \ldots, N\}$, using only knowledge of agent model, i.e. $(A, B, C)$, of the form:

$$
\left\{\begin{aligned}
\dot{x}_{c, i} & =A_{c} x_{c, i}+B_{c} \sigma\left(u_{i}\right)+C_{c} \bar{\zeta}_{i}+D_{c} \hat{\zeta}_{i}, \\
u_{i} & =F_{c} x_{c, i}
\end{aligned}\right.
$$

where $\hat{\zeta}_{i}$ is defined in (4) with $\xi_{i}=H_{c} x_{i, c}$, and $x_{c, i} \in \mathbb{R}^{n_{c}}$, such that regulated state synchronization

$$
\lim _{t \rightarrow \infty}\left(x_{i}-x_{j}\right)=0 \quad \text { for all } i, j \in 1, \ldots, N
$$

is achieved for any $N$ and any graph $\mathcal{G} \in \mathbb{G}_{\mathscr{C}}^{N}$, and for all initial conditions of the agents $x_{i}(0) \in \mathbb{R}^{n}$, all initial conditions of the exosystem $x_{r}(0) \in \mathbb{R}^{n}$, and all initial conditions of the protocols $x_{c, i}(0) \in \mathbb{R}^{n_{c}}$.

Remark 2 In the case offull-state coupling, matrix $C=I$ and we refer to Problem 1 as scalable global regulated state synchronization problem based on localized information exchange for MAS with full-state coupling. 


\section{MAS with Neutrally Stable Agents}

In this section, we will consider the scalable global regulated state synchronization problem for a MAS consisting of neutrally stable agents with input saturation for both networks with full- and partial-state coupling. We make the following assumption on agent models.

Assumption 1 We assume that $(A, B, C)$ is controllable and observable. Moreover, A is neutrally stable, i.e., all the eigenvalues of $A$ are in the closed left half plane and those eigenvalues on the imaginary axis, if any, are semi-simple.

\subsection{Full-state coupling}

In this subsection we consider MAS with full-state coupling.

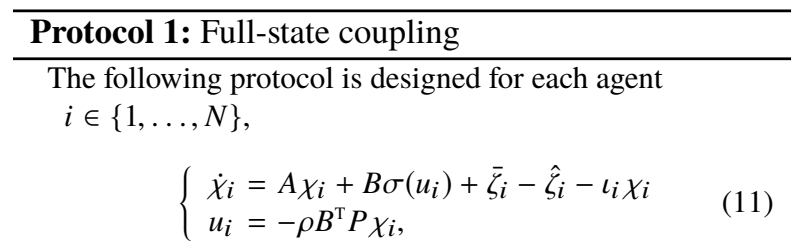

where $\rho>0$ is a parameter with arbitrary positive value and $P>0$ satisfies

$$
P A+A^{\mathrm{T}} P \leqslant 0
$$

since $A$ satisfies Assumption 1. The agents communicate $\xi_{i}$ which is chosen as $\xi_{i}=\chi_{i}$, therefore each agent has access to the following information:

$$
\hat{\zeta}_{i}=\sum_{j=1}^{N} a_{i j}\left(\chi_{i}-\chi_{j}\right)
$$

while $\bar{\zeta}_{i}$ is defined by (8).

We have following theorem for scalable global regulated state synchronization based on localized information exchange for MAS with full-state coupling and neutrally stable agent models.

Theorem 1 Consider a MAS with neutrally stable agents described by (1) where $C=I$, satisfying Assumption 1, and the associated exosystem (5). Let a set of nodes $\mathscr{C}$ be given which defines the set $\mathbb{G}_{\mathscr{C}}^{N}$. Let the associated network communication be given by (8).

Then, the scalable global regulated state synchronization problem based on localized information exchange for MAS with full-state coupling as stated in Problem 1 is solvable. In particular, for any given $\rho>0$, the dynamic protocol (11) solves the regulated state synchronization problem for any $N$ and any graph $G \in \mathbb{G}_{\mathscr{C}}^{N}$.

Proof of Theorem 1: Firstly, by defining $\tilde{x}_{i}=x_{i}-x_{r}$ and $e_{i}=\tilde{x}_{i}-\chi_{i}$ we have

$$
\begin{aligned}
\dot{\tilde{x}}_{i} & =A \tilde{x}_{i}+B \sigma\left(u_{i}\right), \\
\dot{e}_{i} & =A e_{i}-\sum_{j=1}^{N} \bar{\ell}_{i j} e_{j}, \\
u_{i} & =-\rho B^{\mathrm{T}} P\left(\tilde{x}_{i}-e_{i}\right)
\end{aligned}
$$

Then, let

$$
\tilde{x}=\left(\begin{array}{c}
\tilde{x}_{1} \\
\vdots \\
\tilde{x}_{N}
\end{array}\right), u=\left(\begin{array}{c}
u_{1} \\
\vdots \\
u_{N}
\end{array}\right), e=\left(\begin{array}{c}
e_{1} \\
\vdots \\
e_{N}
\end{array}\right) \text {, and } \sigma(u)=\left(\begin{array}{c}
\sigma\left(u_{1}\right) \\
\vdots \\
\sigma\left(u_{N}\right)
\end{array}\right)
$$

then we have the following closed-loop system

$$
\left\{\begin{array}{l}
\dot{\tilde{x}}=(I \otimes A) \tilde{x}+(I \otimes B) \sigma(u), \\
\dot{e}=(I \otimes A-\bar{L} \otimes I) e, \\
u=-\rho\left(I \otimes B^{\mathrm{T}} P\right)(\tilde{x}-e) .
\end{array}\right.
$$

Since all eigenvalues of $\bar{L}$ have positive real part, we have

$$
(T \otimes I)(I \otimes A-\bar{L} \otimes I)\left(T^{-1} \otimes I\right)=I \otimes A-\bar{J} \otimes I
$$

for a non-singular transformation matrix $T$, where (15) is upper triangular Jordan form with $A-\lambda_{i} I$ for $i=1, \cdots, N$ on the diagonal. Since the agents are neutrally stable, i.e. all eigenvalues of $A$ are in the closed left half plane, $A-\lambda_{i} I$ is stable. Therefore, all eigenvalues of $I \otimes A-\bar{L} \otimes I$ have negative real part.

Then, we choose the following Lyapunov function

$$
V=\tilde{x}^{\mathrm{T}}(I \otimes P) \tilde{x}+e^{\mathrm{T}} \bar{P} e
$$

where $P>0$ satisfies condition (12) and $\bar{P}>0$ satisfies $\bar{P}(I \otimes A-\bar{L} \otimes I)+(I \otimes A-\bar{L} \otimes I)^{\mathrm{T}} \bar{P} \leqslant-\left(1+\rho\left\|B^{\mathrm{T}} P\right\|^{2}\right) I$

Thus, we have

$$
\begin{aligned}
\frac{d V}{d t}= & \tilde{x}^{\mathrm{T}} I \otimes\left(P A+A^{\mathrm{T}} P\right) \tilde{x}+2 \tilde{x}^{\mathrm{T}}(I \otimes P B) \sigma(u) \\
& +e^{\mathrm{T}}\left[\bar{P}(I \otimes A-\bar{L} \otimes I)+(I \otimes A-\bar{L} \otimes I)^{\mathrm{T}} \bar{P}\right] e \\
\leqslant & -2 \rho^{-1} u^{\mathrm{T}} \sigma(u)+2 e^{\mathrm{T}}(I \otimes P B) \sigma(u) \\
& -\left(1+\rho\left\|B^{\mathrm{T}} P\right\|^{2}\right) e^{\mathrm{T}} e \\
\leqslant & -2 \rho^{-1} u^{\mathrm{T}} \sigma(u)+\rho^{-1} \sigma^{\mathrm{T}}(u) \sigma(u)-\|e\|^{2}
\end{aligned}
$$

Since $u_{i}^{k} \sigma\left(u_{i}^{k}\right)=\left|u_{i}^{k}\right|\left|\sigma\left(u_{i}^{k}\right)\right| \geqslant\left|\sigma\left(u_{i}^{k}\right)\right|^{2}\left(u_{i}^{k}\right.$ is $k$ th element of $\left.u_{i}, k=1, \cdots, n\right)$, we have $-2 u^{\mathrm{T}} \sigma(u)+$ $\sigma^{\mathrm{T}}(u) \sigma(u) \leqslant 0$. Thus, we obtain $\frac{d V}{d t} \leqslant 0$. 
Meanwhile, we note that $\frac{d V}{d t}=0$ when $I \otimes(P A+$ $\left.A^{\mathrm{T}} P\right) \tilde{x}=0,\left(I \otimes B^{\mathrm{T}} P\right) \tilde{x}=0$, and $e=0$ based on (17). Thus in this case, $\tilde{x}$ is the solution of the dynamics $\dot{\tilde{x}}=$ $(I \otimes A) \tilde{x}$.

Let $S$ be a matrix such that $A+B S$ is Hurwitz stable. Then we have

$(I \otimes P) \dot{\tilde{x}}=I \otimes\left(P A-S^{\mathrm{T}} B^{\mathrm{T}} P\right) \tilde{x}=-I \otimes\left(A^{\mathrm{T}}+S^{\mathrm{T}} B^{\mathrm{T}}\right)(I \otimes P) \tilde{x}$

since $[I \otimes(P A)] \tilde{x}=-\left[I \otimes\left(A^{\mathrm{T}} P\right)\right] \tilde{x}$ and $\left[I \otimes\left(S^{\mathrm{T}} B^{\mathrm{T}} P\right)\right] \tilde{x}=0$. Because $A^{\mathrm{T}}+S^{\mathrm{T}} B^{\mathrm{T}}$ is Hurwitz stable, we have $(I \otimes P) \tilde{x}$ is exponentially growing which contradicts with $\dot{\tilde{x}}=(I \otimes$ A) $\tilde{x}$. It means that $\tilde{x}=0$ is the solution of the above dynamics when $P>0$. Thus, the invariance set $\{(\tilde{x}, e)$ : $\dot{V}(\tilde{x}, e)=0\}$ contains no trajectory of the system except the trivial trajectory $(\tilde{x}, e)=(0,0)$. Therefore, system (14) is globally asymptotically stable based on LaSalle's invariance principle. It means we have $\tilde{x} \rightarrow 0$ and $e \rightarrow 0$ when $t \rightarrow \infty$. Thus we obtain $x_{i} \rightarrow x_{r}$ as $t \rightarrow \infty$, which proves our result.

\subsection{Partial-state coupling}

In this subsection we consider MAS with partial-state coupling.

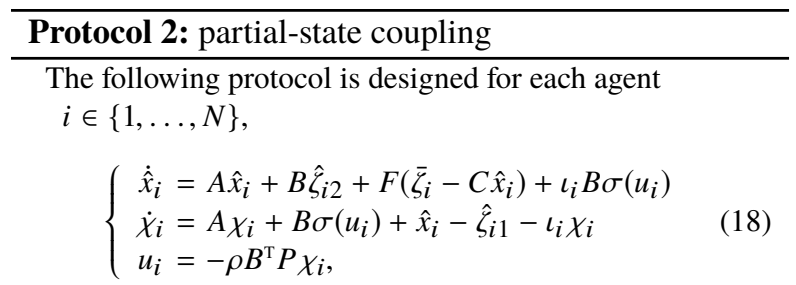

where $F$ is a design matrix such that $A-F C$ is Hurwitz stable, $\rho>0$ is a parameter with arbitrary positive value, and $P$ satisfies (12). In this protocol, the agents

communicate $\xi_{i}=\left(\begin{array}{ll}\xi_{i 1}^{\mathrm{T}}, & \xi_{i 2}^{\mathrm{T}}\end{array}\right)^{\mathrm{T}}=\left(\begin{array}{ll}\chi_{i}^{\mathrm{T}}, & \sigma^{\mathrm{T}}\left(u_{i}\right)\end{array}\right)^{\mathrm{T}}$, i.e.

each agent has access to localized information

$\hat{\zeta}_{i}=\left(\hat{\zeta}_{i 1}^{\mathrm{T}}, \quad \hat{\zeta}_{i 2}^{\mathrm{T}}\right)^{\mathrm{T}}$, where $\hat{\zeta}_{i 1}$ and $\hat{\zeta}_{i 2}$ are defined as

$$
\hat{\zeta}_{i 1}=\sum_{j=1}^{N} a_{i j}\left(\chi_{i}-\chi_{j}\right)
$$

and

$$
\hat{\zeta}_{i 2}=\sum_{j=1}^{N} a_{i j}\left(\sigma\left(u_{i}\right)-\sigma\left(u_{j}\right)\right)
$$

while $\bar{\zeta}_{i}$ is defined via (8).

Then, we have the following theorem for scalable global regulated state synchronization based on local- ized information exchange for MAS with partial-state coupling and neutrally stable agent models.

Theorem 2 Consider a MAS with neutrally stable agents described by (1) satisfying Assumption 1, and the associated exosystem (5). Let a set of nodes $\mathscr{C}$ be given which defines the set $\mathbb{G}_{\mathscr{C}}^{N}$. Let the associated network communication be given by (8).

Then, the scalable global regulated state synchronization problem based on localized information exchange for MAS with partial-state coupling as stated in Problem 1 is solvable. In particular, for any given $\rho>0$, the dynamic protocol (18) solves the scalable regulated state synchronization problem for any $N$ and any graph $\mathcal{G} \in \mathbb{G}_{\mathscr{C}}^{N}$.

Proof of Theorem 2: Similar to the proof of Theorem 1, we have the matrix expression of closed-loop system

$$
\left\{\begin{array}{l}
\dot{\tilde{x}}=(I \otimes A) \tilde{x}+(I \otimes B) \sigma(u) \\
\dot{e}=(I \otimes A-\bar{L} \otimes I) e+\bar{e} \\
\dot{\bar{e}}=I \otimes(A-F C) \bar{e} \\
u=-\rho\left(I \otimes B^{\mathrm{T}} P\right)(\tilde{x}-e)
\end{array}\right.
$$

by $e=\tilde{x}-\chi$, and $\bar{e}=(\bar{L} \otimes I) \tilde{x}-\hat{x}$.

Then, choose the following Lyapunov function

$$
V=\tilde{x}^{\mathrm{T}}(I \otimes P) \tilde{x}+\left(\begin{array}{l}
e \\
\bar{e}
\end{array}\right)^{\mathrm{T}} \tilde{P}\left(\begin{array}{l}
e \\
\bar{e}
\end{array}\right)
$$

where $P>0$ satisfies (12) and $\tilde{P}>0$ satisfies

$$
\tilde{P} \bar{A}+\bar{A}^{\mathrm{T}} \tilde{P} \leqslant-\left(\rho\left\|B^{\mathrm{T}} P\right\|^{2}+1\right) I
$$

with

$$
\bar{A}=\left(\begin{array}{cc}
I \otimes A-\bar{L} \otimes I & I \\
0 & I \otimes(A-F C)
\end{array}\right) .
$$

Similar to Theorem 1, we can obtain the synchronization result $x_{i} \rightarrow x_{r}$ as $t \rightarrow \infty$.

\section{MAS with Double-integrator Agents}

In this section, we will consider scalable global regulated state synchronization problem for MAS consisting of double-integrator agents with input saturation for both networks with full and partial-state coupling.

\subsection{Full-state coupling}

In this subsection, we design dynamic protocols for MAS with full-state coupling and double-integrator agent 
models. First, for agents (1) with double integrator models, we have

$$
A=\left(\begin{array}{cc}
0 & I_{m} \\
0 & 0
\end{array}\right), B=\left(\begin{array}{c}
0 \\
I_{m}
\end{array}\right)
$$

where $A \in \mathbb{R}^{2 m \times 2 m}$ and $B \in \mathbb{R}^{2 m \times m}$. Then, we choose matrix $K=\left(\begin{array}{ll}K_{1} & K_{2}\end{array}\right)$ such that $K_{i} \in \mathbb{R}^{m \times m}, i=1,2$ are arbitrary negative definite matrices.

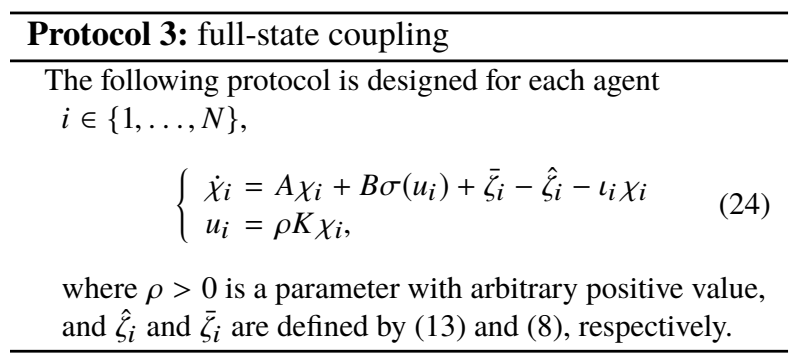

We have the following theorem for scalable global regulated state synchronization problem based on localized information exchange for MAS with full-state coupling and double-integrator agent models.

Theorem 3 Consider a MAS described by (1) with (23) and $C=I$, and the associated exosystem (5). Let a set of nodes $\mathscr{C}$ be given which defines the set $\mathbb{G}_{\mathscr{C}}^{N}$. Let the associated network communication be given by (8).

Then, the scalable global regulated state synchronization problem based on localized information exchange for MAS with full-state coupling as stated in Problem 1 is solvable. In particular, for any given $\rho>0$, the dynamic protocol (24) solves the regulated state synchronization problem for any $N$ and any graph $\mathcal{G} \in \mathbb{G}_{\mathscr{C}}^{N}$.

Proof of Theorem 3: Firstly, similar to Theorem 1, we have

$$
\begin{aligned}
\dot{\tilde{x}}_{i} & =A \tilde{x}_{i}+B \sigma\left(u_{i}\right), \\
\dot{e}_{i} & =A e_{i}-\sum_{j=1}^{N} \bar{\ell}_{i j} e_{j}, \\
u_{i} & =\rho K\left(\tilde{x}_{i}-e_{i}\right)
\end{aligned}
$$

by $\tilde{x}_{i}=x_{i}-x_{r}$ and $e_{i}=\tilde{x}_{i}-\chi_{i}$. Then, let

$$
\tilde{x}=\left(\begin{array}{c}
\tilde{x}_{1} \\
\vdots \\
\tilde{x}_{N}
\end{array}\right), u=\left(\begin{array}{c}
u_{1} \\
\vdots \\
u_{N}
\end{array}\right), e=\left(\begin{array}{c}
e_{1} \\
\vdots \\
e_{N}
\end{array}\right) \text {, and } \sigma(u)=\left(\begin{array}{c}
\sigma\left(u_{1}\right) \\
\vdots \\
\sigma\left(u_{N}\right)
\end{array}\right)
$$

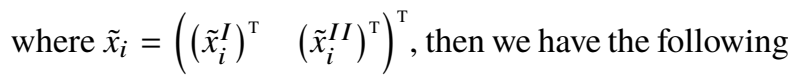

closed-loop system

$$
\left\{\begin{array}{l}
\dot{\tilde{x}}=(I \otimes A) \tilde{x}+(I \otimes B) \sigma(u), \\
\dot{e}=(I \otimes A-\bar{L} \otimes I) e, \\
u=\rho(I \otimes K)(\tilde{x}-e) .
\end{array}\right.
$$

Then, consider the following Lyapunov function

$$
V=\rho \tilde{x}^{\mathrm{T}} I \otimes\left(\begin{array}{cc}
0 & 0 \\
0 & P_{d}
\end{array}\right) \tilde{x}+e^{\mathrm{T}} P_{D} e+2 \int_{0}^{u} \sigma(s) d s
$$

where $P_{d}=-K_{1}$ and $P_{D}>0$ satisfies

$$
P_{D}(I \otimes A-\bar{L} \otimes I)+(I \otimes A-\bar{L} \otimes I)^{\mathrm{T}} P_{D} \leqslant-\gamma I
$$

with $\gamma=1+\rho \varepsilon^{-1}\|K\|^{2}\|I \otimes \tilde{A}-\bar{L} \otimes I\|^{2}$, where $\varepsilon$ is such that $K_{2}<-\frac{\varepsilon}{2} I$ which follows from the choice of $K_{2}$ as negative definite matrix. Note that it can be shown that $V$ is positive definite, i.e. $V>0$ except for $(\tilde{x}, e)=0$ when $V=0$. Then, we have

$$
\begin{aligned}
& \frac{d V}{d t}=2 \rho \sigma^{\mathrm{T}}(u) I \otimes\left[K A+\left(\begin{array}{ll}
0 & P_{d}
\end{array}\right)\right] \tilde{x} \\
& +e^{\mathrm{T}}\left[P_{D}(I \otimes A-\bar{L} \otimes I)+(I \otimes A-\bar{L} \otimes I)^{\mathrm{T}} P_{D}\right] e \\
& +\rho \sigma^{\mathrm{T}}(u) I \otimes\left(K B+B^{\mathrm{T}} K^{\mathrm{T}}\right) \sigma(u) \\
& -2 \rho \sigma^{\mathrm{T}}(u)(I \otimes K)(I \otimes A-\bar{L} \otimes I) e \\
& \leqslant 2 \rho \sigma^{\mathrm{T}}(u) I \otimes\left[K A+\left(\begin{array}{ll}
0 & P_{d}
\end{array}\right)\right] \tilde{x} \\
& -\left(\gamma-\rho \varepsilon^{-1}\|K\|^{2}\|I \otimes \tilde{A}-\bar{L} \otimes I\|^{2}\right)\|e\|^{2} \\
& +\rho \sigma^{\mathrm{T}}(u) I \otimes\left(K B+B^{\mathrm{T}} K^{\mathrm{T}}+\varepsilon I\right) \sigma(u)
\end{aligned}
$$

Meanwhile, we have

$$
K A+\left(\begin{array}{ll}
0 & P_{d}
\end{array}\right)=\left(\begin{array}{ll}
0 & K_{1}
\end{array}\right)+\left(\begin{array}{ll}
0 & P_{d}
\end{array}\right)=0
$$

and $K_{2}<-\frac{\varepsilon}{2} I$ such that

$$
\frac{d V}{d t} \leqslant-\|e\|^{2}+\rho \sigma^{\mathrm{T}}(u) I \otimes\left(K B+B^{\mathrm{T}} K^{\mathrm{T}}+\varepsilon I\right) \sigma(u) \leqslant 0
$$

Meanwhile, we can note that $\frac{d V}{d t}=0$ when $(I \otimes K) \tilde{x}=0$ and $e=0$ since (27). Thus in this case, $\tilde{x}$ is the solution of the dynamics $\dot{\tilde{x}}^{I}=\tilde{x}^{I I}$ and $\dot{\tilde{x}}^{I I}=0$. And then we have $\tilde{x}^{I}=\tilde{x}^{I}\left(t_{0}\right)+t \tilde{x}^{I I}\left(t_{0}\right)$ and $\tilde{x}^{I I}=$ $\tilde{x}^{I I}\left(t_{0}\right)$ with $\tilde{x}^{I}=\left(\begin{array}{lll}\left(\tilde{x}_{1}^{I}\right)^{\mathrm{T}} & \cdots & \left(\tilde{x}_{N}^{I}\right)^{\mathrm{T}}\end{array}\right)^{\mathrm{T}}$ and $\tilde{x}^{I I}=$

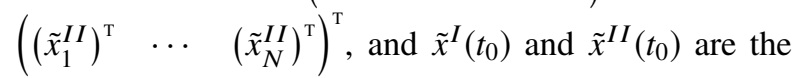
initial value of $\tilde{x}$ at $t_{0}$.

Thus, from $(I \otimes K) \tilde{x}=0$ we obtain

$$
\begin{aligned}
& (I \otimes K) \tilde{x} \\
& =\left[\left(I \otimes K_{1}\right)\left(\tilde{x}^{I}\left(t_{0}\right)+t \tilde{x}^{I I}\left(t_{0}\right)\right) \quad\left(I \otimes K_{2}\right) \tilde{x}^{I I}\left(t_{0}\right)\right]=0
\end{aligned}
$$

i.e. $\left(I \otimes K_{1}\right)\left(\tilde{x}^{I}\left(t_{0}\right)+t \tilde{x}^{I I}\left(t_{0}\right)\right)=0$ and $\left(I \otimes K_{2}\right) \tilde{x}^{I I}\left(t_{0}\right)=$ 0 . Since $K_{1}$ and $K_{2}$ negative definite, we can obtain 
$\tilde{x}^{I}\left(t_{0}\right)=\tilde{x}^{I I}\left(t_{0}\right)=0$. Thus, the invariance set $\{(\tilde{x}, e):$ $\dot{V}(\tilde{x}, e)=0\}$ contains no trajectory of the system except the trivial trajectory $(\tilde{x}, e)=(0,0)$. Therefore, system (25) is globally asymptotically stable based on LaSalle's invariance principle. It means we have $\tilde{x} \rightarrow 0$ and $e \rightarrow 0$ when $t \rightarrow \infty$. Thus we obtain $x_{i} \rightarrow x_{r}$ as $t \rightarrow \infty$, which prove our result.

\subsection{Partial-state coupling}

In this subsection we consider MAS with partial-state coupling.

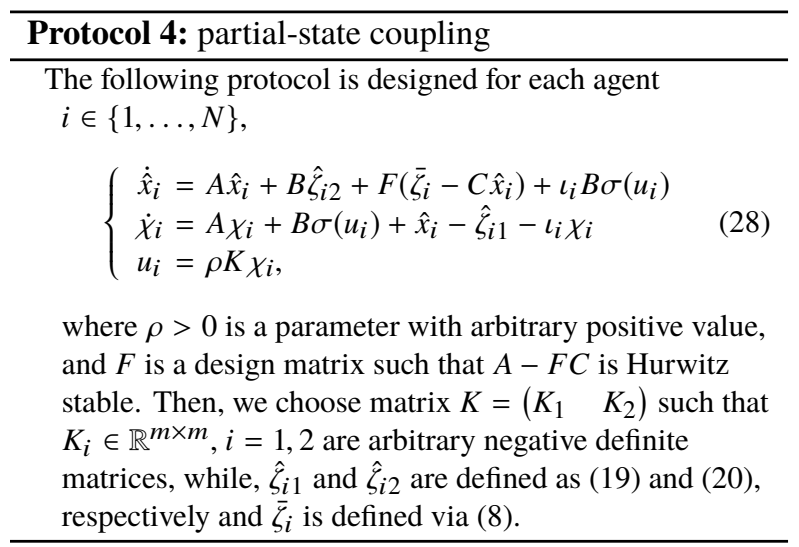

We have the following theorem for scalable global regulated state synchronization problem based on localized information exchange for MAS with partial-state coupling and double-integrator agent models.

Theorem 4 Consider a MAS described by (1), with (23) and $(A, C)$ observable, and the associated exosystem (5). Let a set of nodes $\mathscr{C}$ be given which defines the set $\mathbb{G}_{\mathscr{C}}^{N}$. Let the associated network communication be given by (8).

Then, the scalable global regulated state synchronization problem based on localized information exchange as stated in Problem 1 is solvable. In particular, for any given $\rho>0$, the dynamic protocol (28) solves the scalable regulated state synchronization problem for any $N$ and any graph $\mathcal{G} \in \mathbb{G}_{\mathscr{C}}^{N}$.

Proof of Theorem 4: Similar to Theorem 3, by defining $\tilde{x}_{i}=x_{i}-x_{r}, e=\tilde{x}-\chi$, and $\bar{e}=(\bar{L} \otimes I) \tilde{x}-\hat{x}$, we have the matrix expression of closed-loop system

$$
\begin{aligned}
& \dot{\tilde{x}}=(I \otimes A) \tilde{x}+(I \otimes B) \sigma(u) \\
& \dot{e}=(I \otimes A-\bar{L} \otimes I) e+\bar{e} \\
& \dot{\bar{e}}=I \otimes(A-F C) \bar{e} \\
& u=\rho(I \otimes K)(\tilde{x}-e)
\end{aligned}
$$

Then we choose the following Lyapunov function:

$$
V=\rho \tilde{x}^{\mathrm{T}} I \otimes\left(\begin{array}{cc}
0 & 0 \\
0 & P_{d}
\end{array}\right) \tilde{x}+\left(\begin{array}{l}
e \\
\bar{e}
\end{array}\right)^{\mathrm{T}} P_{D}\left(\begin{array}{l}
e \\
\bar{e}
\end{array}\right)+2 \int_{0}^{u} \sigma(s) d s
$$

where $P_{d}=-K_{1}$ and $P_{D}>0$ satisfies

$$
P_{D} \bar{A}+\bar{A}^{\mathrm{T}} P_{D} \leqslant-\gamma I
$$

where $\gamma=1+\rho \varepsilon^{-1}\|K\|^{2}\|I \otimes A-\bar{L} \otimes I\|^{2}$, and $\varepsilon$ is defined in the proof of Theorem 3 , and $\bar{A}$ is defined in the proof of Theorem 2.

Similar to the proof of Theorem 3, the synchronization result can be obtained.

\section{MAS with Mixed-case Agents}

In this section, we will consider scalable global regulated state synchronization problem via for MAS with agent models mixed-case agents, in presence of input saturation for both networks with full and partial-state coupling. In the following assumption, we consider a class of systems which are introduced in [20].

Assumption 2 We assume that $(A, B, C)$ is controllable and observable. Moreover, A has eigenvalue zero with geometric multiplicity $m$ and algebraic multiplicity $m+q$ with no Jordan blocks of size larger than 2 while the remaining eigenvalues are simple purely imaginary eigenvalues.

Obviously, this class of systems includes the neutrally stable dynamics, single- and double-integrator systems.

\subsection{Full-state coupling}

In this subsection, we design dynamic protocols for each agent via the following steps stated in Protocol 5. 


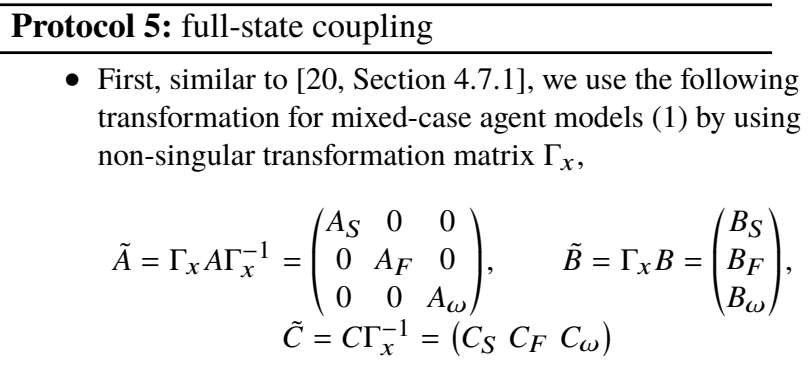

where

$$
A_{S}=\left(\begin{array}{ll}
0 & I \\
0 & 0
\end{array}\right), \quad A_{F}=0, \quad A_{\omega}+A_{\omega}^{\mathrm{T}}=0
$$

- We choose matrix $K$ so that

$$
\begin{gathered}
K \tilde{A}+\tilde{B}^{\mathrm{T}} \Lambda=0 \\
K \tilde{B}+\tilde{B}^{\mathrm{T}} K^{\mathrm{T}}<0
\end{gathered}
$$

with

$$
\Lambda=\left(\begin{array}{ccc}
\Lambda_{0} & 0 & 0 \\
0 & 0 & 0 \\
0 & 0 & I
\end{array}\right) \text { and } \Lambda_{0}=\left(\begin{array}{cc}
0 & 0 \\
0 & P_{d}
\end{array}\right)
$$

where $P_{d}>0$ is any positive definite matrix. The existence of matrix $K$ is proved in [20, Page 235].

- Next, the following protocol is designed for each agent $i \in\{1, \ldots, N\}$,

$$
\left\{\begin{aligned}
\dot{\chi}_{i} & =A \chi_{i}+B \sigma\left(u_{i}\right)+\bar{\zeta}_{i}-\hat{\zeta}_{i}-\iota_{i} \chi_{i} \\
u_{i} & =\rho K \Gamma_{x} \chi_{i}
\end{aligned}\right.
$$

where $\rho>0$ is a parameter with arbitrary positive value, $\hat{\zeta}_{i}$ and $\bar{\zeta}_{i}$ are defined by (13) and (8), respectively.

We have the following theorem for scalable global regulated state synchronization problem based on localized information exchange for MAS with full-state coupling and mixed-case agent models.

Theorem 5 Consider a MAS described by (1) with C = I satisfying Assumption 2, and the associated exosystem (5). Let a set of nodes $\mathscr{C}$ be given which defines the set $\mathbb{G}_{\mathscr{C}}^{N}$. Let the associated network communication be given by (8).

Then, the scalable global regulated state synchronization problem based on localized information exchange for MAS with full-state coupling as stated in Problem 1 is solvable. In particular, for any given $\rho>0$, the dynamic protocol (34) with (32) and (33) solves the regulated state synchronization problem for any $N$ and any graph $\mathcal{G} \in \mathbb{G}_{\mathscr{C}}^{N}$.
Proof of Theorem 5: Firstly, we have

$$
\begin{aligned}
\dot{\tilde{x}}_{i} & =A \tilde{x}_{i}+B \sigma\left(u_{i}\right), \\
\dot{e}_{i} & =A e_{i}-\sum_{j=1}^{N} \bar{\ell}_{i j} e_{j}, \\
u_{i} & =\rho K \Gamma_{x}\left(\tilde{x}_{i}-e_{i}\right)
\end{aligned}
$$

by $\tilde{x}_{i}=x_{i}-x_{r}$ and $e_{i}=\tilde{x}_{i}-\chi_{i}$. Then, let

$$
\tilde{x}=\left(\begin{array}{c}
\tilde{x}_{1} \\
\vdots \\
\tilde{x}_{N}
\end{array}\right), u=\left(\begin{array}{c}
u_{1} \\
\vdots \\
u_{N}
\end{array}\right), e=\left(\begin{array}{c}
e_{1} \\
\vdots \\
e_{N}
\end{array}\right) \text {, and } \sigma(u)=\left(\begin{array}{c}
\sigma\left(u_{1}\right) \\
\vdots \\
\sigma\left(u_{N}\right)
\end{array}\right)
$$

then we have the following closed-loop system

$$
\left\{\begin{array}{l}
\dot{\tilde{x}}=(I \otimes A) \tilde{x}+(I \otimes B) \sigma(u), \\
\dot{e}=(I \otimes A-\bar{L} \otimes I) e \\
u=\rho I \otimes K \Gamma_{x}(\tilde{x}-e) .
\end{array}\right.
$$

We transform mixed-case agent model (35) as

$$
\left\{\begin{array}{l}
\dot{\eta}=\left(\begin{array}{cc}
I \otimes \tilde{A} & 0 \\
0 & I \otimes \tilde{A}-\bar{L} \otimes I
\end{array}\right) \eta+\left(\begin{array}{c}
I \otimes \tilde{B} \\
0
\end{array}\right) \sigma(u) \\
u=\rho I \otimes(K-K) \eta
\end{array}\right.
$$

by a non-singular matrix $I \otimes \Gamma_{x}$, where $\eta=\left(\begin{array}{ll}\eta_{x}^{\mathrm{T}} & \eta_{e}^{\mathrm{T}}\end{array}\right)^{\mathrm{T}}=$ $\left(\left(I \otimes \Gamma_{x}^{\mathrm{T}}\right) \tilde{x}^{\mathrm{T}} \quad\left(I \otimes \Gamma_{x}^{\mathrm{T}}\right) e^{\mathrm{T}}\right)^{\mathrm{T}}$.

Next, we choose the following Lyapunov function:

$$
V=\eta^{\mathrm{T}}\left(\begin{array}{cc}
\rho I \otimes \Lambda & 0 \\
0 & P_{0}
\end{array}\right) \eta+2 \int_{0}^{u} \sigma(s) d s
$$

where $P_{0}>0$ satisfies

$$
P_{0}(I \otimes \tilde{A}-\bar{L} \otimes I)+(I \otimes \tilde{A}-\bar{L} \otimes I)^{\mathrm{T}} P_{0} \leqslant-\gamma I
$$

with $\gamma=1+\rho \varepsilon^{-1}\|K\|^{2}\|I \otimes \tilde{A}-\bar{L} \otimes I\|^{2}$, where $\varepsilon$ is such that $K \tilde{B}+\tilde{B}^{\mathrm{T}} K^{\mathrm{T}}<-\varepsilon I$, note that (33) guarantees existence of $\varepsilon$. It can be shown that $V$ is positive definite, i.e. $V>0$ except for $(\tilde{x}, e)=0$ when $V=0$. Then, we have

$$
\begin{aligned}
\frac{d V}{d t}= & 2 \eta^{\mathrm{T}}\left(\begin{array}{cc}
\rho I \otimes(\Lambda \tilde{A}) & 0 \\
0 & P_{0}(I \otimes \tilde{A}-\bar{L} \otimes I)
\end{array}\right) \eta \\
& +2 \rho \eta^{\mathrm{T}}\left(\begin{array}{c}
I \otimes(\Lambda \tilde{B}) \\
0
\end{array}\right) \sigma(u) \\
& +2 \rho \sigma^{\mathrm{T}}(u)(I \otimes(K \tilde{A}) \quad-(I \otimes K \tilde{A}-\bar{L} \otimes K)) \eta \\
& +2 \rho \sigma^{\mathrm{T}}(u) I \otimes(K \tilde{B}) \sigma(u) \\
\leqslant & -\gamma \eta_{e}^{\mathrm{T}} \eta_{e}+2 \rho \sigma^{\mathrm{T}}(u)(I \otimes K \tilde{B}) \sigma(u) \\
& -2 \rho \sigma^{\mathrm{T}}(u)(I \otimes K)(I \otimes \tilde{A}-\bar{L} \otimes I) \eta_{e} \\
\leqslant & -\gamma \eta_{e}^{\mathrm{T}} \eta_{e}+\varepsilon^{-1} \rho\|K\|^{2}\|I \otimes \tilde{A}-\bar{L} \otimes I\|^{2} \eta_{e}^{\mathrm{T}} \eta_{e} \\
& +\rho \sigma^{\mathrm{T}}(u)\left(I \otimes\left(K \tilde{B}+\tilde{B}^{\mathrm{T}} K^{\mathrm{T}}+\varepsilon I\right)\right) \sigma(u) \\
= & -\left\|\eta_{e}\right\|^{2}+\rho \sigma^{\mathrm{T}}(u)\left[I \otimes\left(K \tilde{B}+\tilde{B}^{\mathrm{T}} K^{\mathrm{T}}+\varepsilon I\right)\right] \sigma(u)
\end{aligned}
$$


since we have (32) and (38). Because $(\tilde{A}, \tilde{B})$ is surjective, we have a solution $K$ such that $\frac{d V}{d t} \leqslant 0$ provided (33).

Then, we note that the $\frac{d V}{d t}=0$ when $(I \otimes K) \eta_{x}=0$ and $\eta_{e}=0$, the dynamics of (36) becomes $\dot{\eta}_{x}=(I \otimes \tilde{A}) \eta_{x}$.

Similar to the proof of [20, Theorem 4.61] with (32) and (33), we can obtain $(I \otimes K) \eta_{x}=0$ only when $\eta_{x}\left(t_{0}\right)=$ 0 .

Thus, we obtain the global asymptotic stability of the closed-loop system (36), i.e. we have $\eta_{x} \rightarrow 0$. It implies $\tilde{x} \rightarrow 0$ since $\left(I \otimes \Gamma_{x}^{-1}\right) \eta_{x} \rightarrow 0$ when $t \rightarrow \infty$, and thus we have $x_{i} \rightarrow x_{r}$ as $t \rightarrow \infty$, which prove our result.

\subsection{Partial-state coupling}

In this subsection we consider MAS with partial-state coupling.

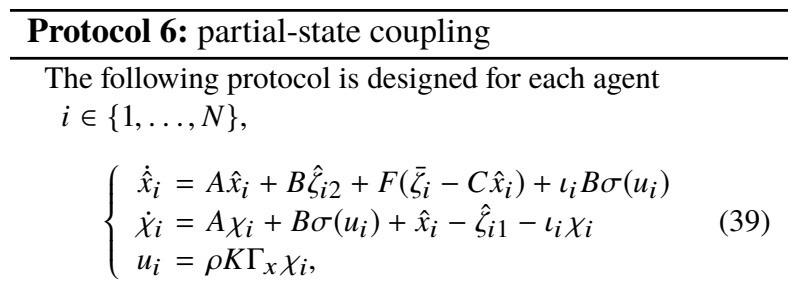

where $F$ is a design matrix such that $A-F C$ is Hurwitz stable, $\Gamma_{x}$ is a non-singular matrix, $\rho>0$ is a parameter with arbitrary positive value, and $K$ satisfies (32) and (33), where $\hat{\zeta}_{i 1}$ and $\hat{\zeta}_{i 2}$ are defined as (19) and (20), respectively and $\bar{\zeta}_{i}$ is defined via (8).

We have the following theorem for scalable global regulated state synchronization problem based on localized information exchange for MAS with partial-state coupling and mixed-case agent models.

Theorem 6 Consider a MAS described by (1) satisfying Assumption 2, and the associated exosystem (5). Let a set of nodes $\mathscr{C}$ be given which defines the set $\mathbb{G}_{\mathscr{C}}^{N}$. Let the associated network communication be given by (8).

Then, the scalable global regulated state synchronization problem based on localized information exchange as stated in Problem 1 is solvable. In particular, for any given $\rho>0$, the dynamic protocol (39) with (32) and (33) solves the scalable regulated state synchronization problem for any $N$ and any graph $G \in \mathbb{G}_{\mathscr{C}}^{N}$.

Proof of Theorem 6: Similar to Theorem 3, by defining $\tilde{x}_{i}=x_{i}-x_{r}, e=\tilde{x}-\chi$, and $\bar{e}=(\bar{L} \otimes I) \tilde{x}-\hat{x}$, we have the matrix expression of closed-loop system

$$
\begin{aligned}
& \dot{\tilde{x}}=(I \otimes A) \tilde{x}+(I \otimes B) \sigma(u) \\
& \dot{e}=(I \otimes A-\bar{L} \otimes I) e+\bar{e} \\
& \dot{\bar{e}}=I \otimes(A-F C) \bar{e} \\
& u=\rho\left(I \otimes K \Gamma_{x}\right)(\tilde{x}-e)
\end{aligned}
$$

Then, by using nonsingular matrix $I \otimes \Gamma_{x}$, we can obtain

$$
\begin{aligned}
& \dot{\eta}_{x}=(I \otimes \tilde{A}) \eta_{x}+(I \otimes \tilde{B}) \sigma(u) \\
& \dot{\eta}_{e}=(I \otimes \tilde{A}-\bar{L} \otimes I) \eta_{e}+\eta_{\bar{e}} \\
& \dot{\eta}_{\bar{e}}=I \otimes\left(\tilde{A}-\Gamma_{x} F \tilde{C}\right) \eta_{\bar{e}} \\
& u=\rho(I \otimes K)\left(\eta_{x}-\eta_{e}\right)
\end{aligned}
$$

where $\eta_{x}=\left(I \otimes \Gamma_{x}\right) \tilde{x}, \eta_{e}=\left(I \otimes \Gamma_{x}\right) e$, and $\eta_{\bar{e}}=\left(I \otimes \Gamma_{x}\right) \bar{e}$.

Then we choose the following Lyapunov function:

$$
V=\bar{\eta}^{\mathrm{T}}\left(\begin{array}{cc}
\rho I \otimes \Lambda & 0 \\
0 & P_{0}
\end{array}\right) \bar{\eta}+2 \int_{0}^{u} \sigma(s) d s
$$

where $\bar{\eta}=\left(\begin{array}{lll}\eta_{x}^{\mathrm{T}} & \eta_{e}^{\mathrm{T}} & \eta_{\bar{e}}^{\mathrm{T}}\end{array}\right)^{\mathrm{T}}$ and $P_{0}>0$ satisfies

$$
P_{0} \hat{A}+\hat{A}^{\mathrm{T}} P_{0} \leqslant-\gamma I
$$

where $\gamma=1+\varepsilon^{-1} \rho\|K\|^{2}\|I \otimes \tilde{A}-\bar{L} \otimes I\|^{2}$, and $\varepsilon$ is the same as in the proof of Theorem 5 and

$$
\hat{A}=\left(\begin{array}{cc}
I \otimes \tilde{A}-\bar{L} \otimes I & I \\
0 & I \otimes\left(\tilde{A}-\Gamma_{x} F \tilde{C}\right)
\end{array}\right) .
$$

Thus, similar to the proof of Theorem 5, the synchronization result can be obtained.

Remark 3 It is worth to note that in all of the protocols for MAS with neutrally stable, double-integrator, and mixed of double-integrator, single-integrator and neutrally stable dynamics, the choice of positive parameter $\rho$ is independent of the communication graph and as such it establishes infinite gain margin for our protocols.

\section{Numerical Example}

In this section, we will illustrate the effectiveness of our protocols with numerical examples for global synchronization of MAS with double-integrator and mixed-case agent models with partial-state coupling.

\section{Example 1: Double-integrator}

Consider a MAS with double-integrators agent models (1) as:

$$
\begin{aligned}
\dot{x}_{i} & =\left(\begin{array}{ll}
0 & 1 \\
0 & 0
\end{array}\right) x_{i}+\left(\begin{array}{l}
0 \\
1
\end{array}\right) \sigma\left(u_{i}\right), \\
y_{i} & =\left(\begin{array}{ll}
1 & 0
\end{array}\right) x_{i}
\end{aligned}
$$

and the exosystem:

$$
\dot{x}_{r}=\left(\begin{array}{ll}
0 & 1 \\
0 & 0
\end{array}\right) x_{r}, \quad y_{r}=\left(\begin{array}{ll}
1 & 0
\end{array}\right) x_{r}
$$




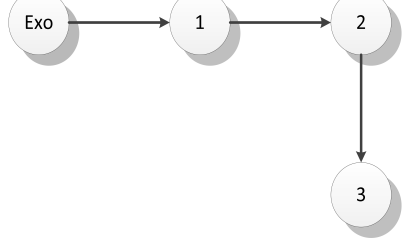

Figure 1: The directed communication network 1

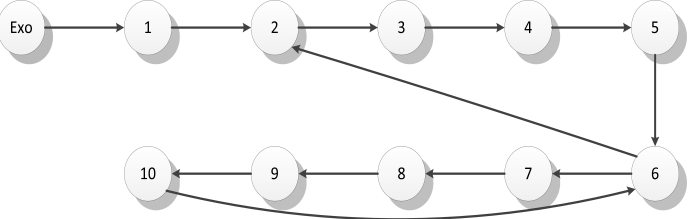

Figure 2: The directed communication network 2

By choosing parameter $\rho=1$ and matrices $F$ and $K$ as

$$
F=\left(\begin{array}{l}
1 \\
2
\end{array}\right), \quad K=\left(\begin{array}{ll}
-10 & -2
\end{array}\right)
$$

the scalable Protocol 4 would be equal to

$$
\left\{\begin{array}{l}
\dot{\hat{x}}_{i}=\left(\begin{array}{ll}
-1 & 1 \\
-2 & 0
\end{array}\right) \hat{x}_{i}+\left(\begin{array}{l}
0 \\
1
\end{array}\right) \hat{\zeta}_{i 2}+\left(\begin{array}{l}
1 \\
2
\end{array}\right) \bar{\zeta}_{i}+\iota_{i}\left(\begin{array}{l}
0 \\
1
\end{array}\right) \sigma\left(u_{i}\right) \\
\dot{\chi}_{i}=\left(\begin{array}{ll}
0 & 1 \\
0 & 0
\end{array}\right) \chi_{i}+\left(\begin{array}{l}
0 \\
1
\end{array}\right) \sigma\left(u_{i}\right)+\hat{x}_{i}-\hat{\zeta}_{i 1}-\iota_{i} \chi_{i} \\
u_{i}=(-10-2) \chi_{i},
\end{array}\right.
$$

where $\iota_{1}=1$ and $\iota_{i}=0$ for $i=\{1, \ldots, N\}$. First, consider a MAS with 3 nodes and communication graph as Figure 1.

To illustrate the scalibility of our protocols we show that the designed protocol will also work for MAS with 10 nodes with communication topology as Figure 2.

The simulation results are shown in Figure 3 and Figure 4 for MAS with 3 and 10 agents, respectively.

\section{Example 2: Mixed-case}

In this example, we consider MAS with mixed-case agent model which contains two double-integrator, one
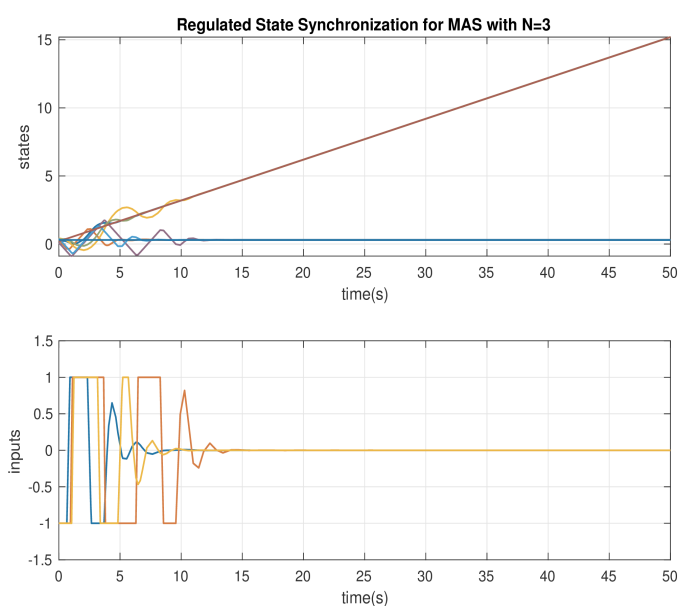

Figure 3: Regulated state synchronization for MAS with double-integrator agents, partial-state coupling and 3 agents

single-integrator and neutrally stable dynamics as:

$$
\begin{aligned}
\dot{x}_{i} & =\left(\begin{array}{lllllll}
0 & 0 & 1 & 0 & 0 & 0 & 0 \\
0 & 0 & 0 & 1 & 0 & 0 & 0 \\
0 & 0 & 0 & 0 & 0 & 0 & 0 \\
0 & 0 & 0 & 0 & 0 & 0 & 0 \\
0 & 0 & 0 & 0 & 0 & 0 & 0 \\
0 & 0 & 0 & 0 & 0 & 0 & 1 \\
0 & 0 & 0 & 0 & 0 & -1 & 0
\end{array}\right) x_{i}+\left(\begin{array}{lll}
0 & 1 & 3 \\
0 & 0 & 5 \\
1 & 2 & 4 \\
0 & 1 & 6 \\
0 & 0 & 1 \\
1 & 1 & 0 \\
1 & 0 & 1
\end{array}\right) \sigma\left(u_{i}\right) \\
y_{i} & =\left(\begin{array}{lllllll}
1 & 1 & 1 & 1 & 1 & 1 & 1 \\
1 & 0 & 0 & 0 & 0 & 0 & 0 \\
0 & 0 & 0 & 0 & 0 & 0 & 1 \\
0 & 0 & 1 & 1 & 1 & 1 & 1
\end{array}\right) x_{i}
\end{aligned}
$$

and the associated exosystem:

$$
\dot{x}_{r}=\left(\begin{array}{ccccccc}
0 & 0 & 1 & 0 & 0 & 0 & 0 \\
0 & 0 & 0 & 1 & 0 & 0 & 0 \\
0 & 0 & 0 & 0 & 0 & 0 & 0 \\
0 & 0 & 0 & 0 & 0 & 0 & 0 \\
0 & 0 & 0 & 0 & 0 & 0 & 0 \\
0 & 0 & 0 & 0 & 0 & 0 & 1 \\
0 & 0 & 0 & 0 & 0 & -1 & 0
\end{array}\right) x_{r}, \quad y_{r}=\left(\begin{array}{lllllll}
1 & 1 & 1 & 1 & 1 & 1 & 1 \\
1 & 0 & 0 & 0 & 0 & 0 & 0 \\
0 & 0 & 0 & 0 & 0 & 0 & 1 \\
0 & 0 & 1 & 1 & 1 & 1 & 1
\end{array}\right) x_{r}
$$

We choose parameter $\rho=1$ and matrix $K$ and $F$ as 

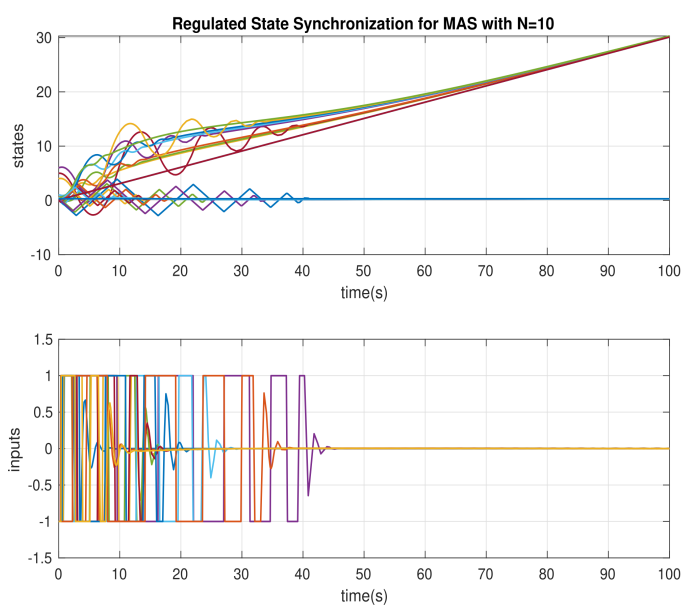

Figure 4: Regulated state synchronization for MAS with double-integrator agents, partial-state coupling and 10 agents
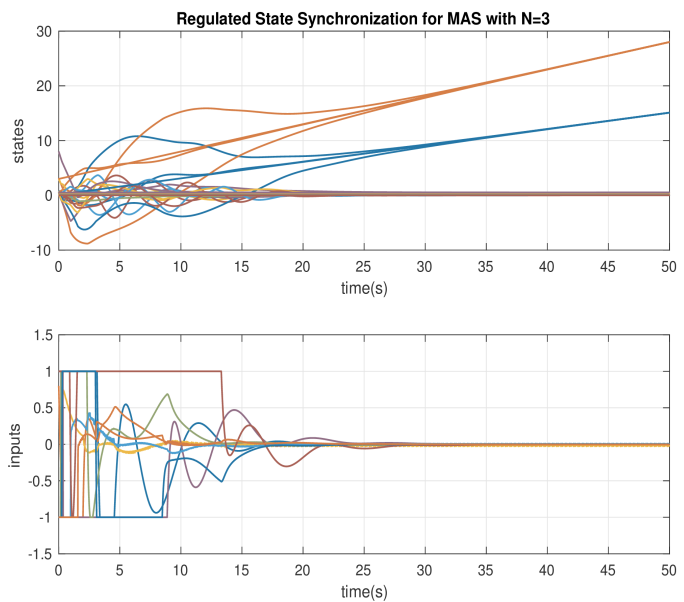

Figure 5: Regulated state synchronization for MAS with mixed-case agents, partial-state coupling and 3 agents

following:

$$
\begin{aligned}
F & =\left(\begin{array}{cccc}
0.55 & 6.81 & 0.73 & -0.42 \\
7.97 & -7.41 & 1.30 & -8.30 \\
0.57 & 10 & 2.97 & 0.37 \\
11.14 & -10.32 & 5.06 & -11.24 \\
-5.92 & -0.92 & 3.66 & 7.89 \\
-7.01 & 1.98 & -14.49 & 8.53 \\
1.35 & -0.27 & 8.48 & -1.52
\end{array}\right) \\
K & =\left(\begin{array}{ccccccc}
-1 & 0 & -4 & 6 & -22 & -1 & 1 \\
-2 & -1 & -3 & -2 & 18 & 0 & 1 \\
-4 & -6 & -5 & -3 & -61 & -1 & 0
\end{array}\right)
\end{aligned}
$$
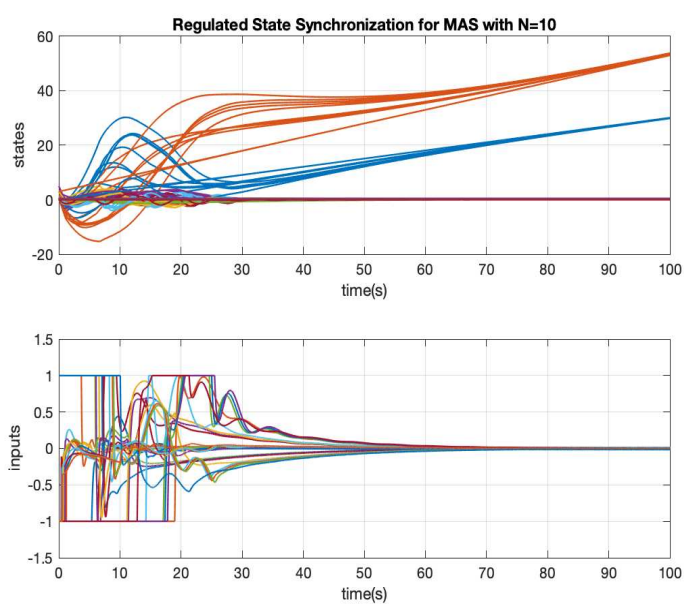

Figure 6: Regulated state synchronization for MAS with mixed-case agents, partial-state coupling and 10 agents

Consider a MAS with 3 agents, and associated directed communication topology shown in Figure 1.

The simulation results for global state synchronization of the MAS with partial-state coupling via scalable dynamic protocol (39) are shown in Figure 5.

To show the scalability of our protocol designs, we consider a MAS with 10 nodes and agent models as the previous case with communication topology as Figure 2.

The simulation results shown in Figure 6 show that global state synchronization is achieved with the same designed protocol.

\section{References}

[1] D. Chowdhury and H. K. Khalil. Synchronization in networks of identical linear systems with reduced information. In American Control Conference, pages 5706-5711, Milwaukee, WI, 2018.

[2] J. Fu, G. Wen, W. Yu, and Z. Ding. Finite-time consensus for second-order multi-agent systems with input saturation. IEEE Trans. Circ. \& Syst.-II Express briefs, 65(11):1758-1762, 2018.

[3] C. Godsil and G. Royle. Algebraic graph theory, volume 207 of Graduate Texts in Mathematics. Springer-Verlag, New York, 2001.

[4] H.F. Grip, T. Yang, A. Saberi, and A.A. Stoorvogel. Output synchronization for heterogeneous networks of nonintrospective agents. Automatica, 48(10):2444-2453, 2012.

[5] H. Kim, H. Shim, J. Back, and J. Seo. Consensus of output-coupled linear multi-agent systems under fast switching network: averaging approach. Automatica, 49(1):267-272, 2013.

[6] H. Kim, H. Shim, and J.H. Seo. Output consensus of heterogeneous uncertain linear multi-agent systems. IEEE 
Trans. Aut. Contr., 56(1):200-206, 2011.

[7] Y. Li, J. Xiang, and W. Wei. Consensus problems for linear time-invariant multi-agent systems with saturation constraints. IET Control Theory and Applications, 5(6):823-829, 2011.

[8] Z. Liu, D. Nojavanzadeh, D. Saberi, A. Saberi, and A. A. Stoorvogel. Regulated state synchronization for discretetime homogeneous networks of non-introspective agents in presence of unknown non-uniform input delays: A scale-free protocol design (with a general matlab implementation). arXiv preprint arXiv:2002.06577, 2020.

[9] Z. Liu, D. Nojavanzadeh, D. Saberi, A. Saberi, and A. A. Stoorvogel. Regulated state synchronization for homogeneous networks of non-introspective agents in presence of input delays: A scale-free protocol design (with a general matlab implementation). arXiv preprint arXiv:2001.02117, 2020.

[10] Z. Liu, A. Saberi, A. A. Stoorvogel, and D. Nojavanzadeh. Global and semi-global regulated state synchronization for homogeneous networks of non-introspective agents in presence of input saturationâĂŞ a scale-free protocol design. In IEEE Conference on Decision and Control (CDC), 2019.

[11] Z. Liu, A. Saberi, A. A. Stoorvogel, and M. Zhang. Passivity-based state synchronization of homogeneous multiagent systems via static protocol in the presence of input saturation. Int. J. Robust \& Nonlinear Control, 28(7):2720-2741, 2018.

[12] Z. Liu, M. Zhang, A. Saberi, and A. A. Stoorvogel. State synchronization of multi-agent systems via static or adaptive nonlinear dynamic protocols. Automatica, 95:316327, 2018.

[13] Z. Liu, M. Zhang, A. Saberi, and A.A. Stoorvogel. Passivity based state synchronization of homogeneous discretetime multi-agent systems via static protocol in the presence of input delay. European Journal of Control, 41:1624, 2018.

[14] Z. Meng, Z. Zhao, and Z. Lin. On global leader-following consensus of identical linear dynamic systems subject to actuator saturation. Syst. \& Contr. Letters, 62(2):132$142,2013$.

[15] D. Nojavanzadeh, Z. Liu, A. Saberi, and A. A. Stoorvogel. Output and regulated output synchronization of heterogeneous multi-agent systems: A scale-free protocol design using no information about communication network and the number of agents. In American Control Conference (ACC), 2020.

[16] R. Olfati-Saber, J.A. Fax, and R.M. Murray. Consensus and cooperation in networked multi-agent systems. Proc. of the IEEE, 95(1):215-233, 2007.

[17] R. Olfati-Saber and R.M. Murray. Consensus problems in networks of agents with switching topology and timedelays. IEEE Trans. Aut. Contr., 49(9):1520-1533, 2004.

[18] W. Ren. On consensus algorithms for double-integrator dynamics. IEEE Trans. Aut. Contr., 53(6):1503-1509, 2008 .
[19] W. Ren and Y.C. Cao. Distributed coordination of multiagent networks. Communications and Control Engineering. Springer-Verlag, London, 2011.

[20] A. Saberi, A.A. Stoorvogel, and P. Sannuti. Internal and external stabilization of linear systems with constraints. Birkhäuser, Boston, MA, 2012.

[21] L. Scardovi and R. Sepulchre. Synchronization in networks of identical linear systems. Automatica, 45(11):2557-2562, 2009.

[22] J.H. Seo, J. Back, H. Kim, and H. Shim. Output feedback consensus for high-order linear systems having uniform ranks under switching topology. IET Control Theory and Applications, 6(8):1118-1124, 2012.

[23] J.H. Seo, H. Shim, and J. Back. Consensus of high-order linear systems using dynamic output feedback compensator: low gain approach. Automatica, 45(11):26592664, 2009.

[24] A. Stoorvogel, A. Saberi, M. Zhang, and Z. Liu. Solvability conditions and design for synchronization of discretetime multi-agent systems. Int. J. Robust \& Nonlinear Control, 28(4):1381-1401, 2018.

[25] A. A. Stoorvogel, A. Saberi, and M. Zhang. Solvability conditions and design for state synchronization of multiagent systems. Automatica, 84:43-47, 2017.

[26] Y. Su and J. Huang. Stability of a class of linear switching systems with applications to two consensus problem. IEEE Trans. Aut. Contr., 57(6):1420-1430, 2012.

[27] S.E. Tuna. LQR-based coupling gain for synchronization of linear systems. Available: arXiv:0801.3390v1, 2008.

[28] S.E. Tuna. Conditions for synchronizability in arrays of coupled linear systems. IEEE Trans. Aut. Contr., 55(10):2416-2420, 2009.

[29] P. Wieland, J.S. Kim, and F. Allgöwer. On topology and dynamics of consensus among linear high-order agents. International Journal of Systems Science, 42(10):18311842, 2011.

[30] P. Wieland, R. Sepulchre, and F. Allgöwer. An internal model principle is necessary and sufficient for linear output synchronization. Automatica, 47(5):1068-1074, 2011.

[31] C.W. Wu. Synchronization in complex networks of nonlinear dynamical systems. World Scientific Publishing Company, Singapore, 2007.

[32] T. Yang, Z. Meng, D.V. Dimarogonas, and K.H. Johansson. Global consensus for discrete-time multi-agent systems with input saturation constraints. Automatica, 50(2):499-506, 2014.

[33] T. Yang, A. Saberi, A.A. Stoorvogel, and H.F. Grip. Output synchronization for heterogeneous networks of introspective right-invertible agents. Int. J. Robust \& Nonlinear Control, 24(13):1821-1844, 2014.

[34] X. Yi, T. Yang, J. Wu, and K. H. Johansson. Distributed event-triggered control for global consensus of multi-agent systems with input saturation. Automatica, 100:1-9, 2019. 